\title{
An ideal Waring problem with restricted summands
}

\author{
by \\ Michael A. Bennett (Waterloo, Ont.)
}

1. Introduction. If we define $g(k)$ to be the order of the set $\left\{1^{k}, 2^{k}, \ldots\right\}$ as an additive basis for the positive integers, then the ideal Waring problem is to show that

$$
g(k)=2^{k}+\left[(3 / 2)^{k}\right]-2
$$

for all $k \in N([x]$ is the integer part of $x)$. By work of Mahler [9], this holds for all but finitely many $k$, but the result is ineffective and does not yield a bound upon these exceptional values. Computations by Kubina and Wunderlich [8], however, have shown (1) to obtain for all $k \leq 471600000$.

We consider representations of positive integers as sums of elements of

$$
S_{N}^{(k)}=\left\{1^{k}, N^{k},(N+1)^{k}, \ldots\right\}
$$

where $N \geq 2$ is an integer. A theorem of Rieger [10] gives that $S_{N}^{(k)}$ forms an additive basis for $\mathbb{N}$ for any natural number $k$. If we let $g_{N}(k)$ denote the order of this basis (so that $g_{2}(k)=g(k)$ ), then the aim of this paper is to prove an analog of (1). To be precise, we have

THEOREM 1.1. If $4 \leq N \leq(k+1)^{(k-1) / k}-1$, then

$$
g_{N}(k)=N^{k}+\left[\left(\frac{N+1}{N}\right)^{k}\right]-2 .
$$

This follows from two results of the author, namely

Theorem 1.2 (Bennett [2]). Suppose $k \geq 6$ and $M \geq e^{446 k^{6}}$ are positive integers. Then there exist $s$ integers $x_{1}, x_{2}, \ldots, x_{s}$, where $s<6 k \log k+$ $(3 \log 6+4) k$, such that $x_{i} \geq M^{1 /\left(8 k^{3}\right)}$ for $i=1,2, \ldots, s$ and

$$
M=x_{1}^{k}+x_{2}^{k}+\ldots+x_{s}^{k} .
$$


Theorem 1.3 (Bennett [3]). Define $\|x\|=\min _{M \in \mathbb{Z}}|x-M|$. If $4 \leq N \leq$ $k \cdot 3^{k}$, then

$$
\left\|\left(\frac{N+1}{N}\right)^{k}\right\|>3^{-k}
$$

The first of these is essentially a slight generalization of Vinogradov's earliest upper bound for $G(k)$ in the standard Waring problem (see [11]). Since its proof entails making only minor modifications to a well known argument (to compensate for the restriction to $k$ th powers of integers $\geq N$ ), we will not duplicate it here. We use this rather old fashioned approach instead of later versions of order $3 k \log k$ or $2 k \log k$ because these induce a lower bound for $M$ which is too large to be practical for our purposes (though they increase the bound for $x_{i}$ ). The difficulty chiefly arises from the size of the implied constant in

$$
\eta(a) \ll q^{\varepsilon}
$$

where $\eta(a)$ is the number of solutions to the congruence

$$
v^{k} \equiv a \bmod q
$$

for $v$ and $a$ integers in $[0, q-1]$.

The second theorem we use is an effective sharpening of a result of Beukers [4] on fractional parts of powers of rationals. It utilizes Padé approximation to the polynomial $(1-z)^{k}$ and some estimates on primes dividing binomial coefficients.

2. Dickson's ascent argument. We adopt the notation

$$
\alpha=\left[\left(\frac{N+1}{N}\right)^{k}\right] \text { and } \beta=(N+1)^{k}-N^{k} \cdot\left[\left(\frac{N+1}{N}\right)^{k}\right] \text {. }
$$

Suppose $N \leq(k+1)^{(k-1) / k}-1$ and write $[a, b] \in S_{N}^{(k)}(m)$ (or $(a, b) \in$ $S_{N}^{(k)}(m)$ ) if every integer in $[a, b]$ (respectively $(a, b)$ ) can be written as a sum of at most $m$ elements of $S_{N}^{(k)}$ (where we allow repetitions). Following Dickson [6], we count the number of elements of $S_{N}(k)$ required for representations of "small" integers before applying an ascent argument to enable the use of Theorem 1.2.

Before we begin, we need a pair of preliminary lemmas.

Lemma 2.1. If $N, k \geq 2$ and $M$ are integers then

$$
(N+1)^{k}-M N^{k}=1
$$

has only the solutions $N=2$ and $k=2$ or 4 .

Proof. Suppose that

$$
(N+1)^{k}=M N^{k}+1
$$


where $N \geq 2$ and $k \geq 2$ (but not $N=k=2$ ). If $k$ is even, then we may write

$$
\left((N+1)^{k / 2}-1\right)\left((N+1)^{k / 2}+1\right)=M N^{k}
$$

and so conclude if $N$ is odd that $N^{k}$ divides $(N+1)^{k / 2}-1$. Since this implies $N^{2}<N+1$, it contradicts $N \geq 2$. If, however, $N$ is even, then we have

$$
N^{k} \mid 2\left((N+1)^{k / 2}-1\right) \quad \text { if } N \equiv 0 \bmod 4
$$

or

$$
N^{k} \mid 2^{k}\left((N+1)^{k / 2}-1\right) \quad \text { if } N \equiv 2 \bmod 4 .
$$

From (4), we have $N^{2}<2(N+1)$, which contradicts $N \equiv 0 \bmod 4$ while (5) implies that $N=2$. Since 3 belongs to the exponent $2^{k-2}$ modulo $2^{k}$, we must have $2^{k-2}$ dividing $k$, so that $k \leq 4$.

It remains only to consider odd $k$. We can write, from (2),

$$
\sum_{i=1}^{k}\left(\begin{array}{l}
k \\
i
\end{array}\right) N^{i}=M N^{k}
$$

and proceed via induction, proving that $\operatorname{ord}_{N}(k) \rightarrow \infty$, thus contradicting any a priori upper bound for $k$. From (6), we clearly have $N \mid k$ and if we suppose that $N^{a} \mid k$, then since

$$
\operatorname{ord}_{p}\left(\begin{array}{c}
k \\
i
\end{array}\right) \geq \operatorname{ord}_{p} k-\operatorname{ord}_{p} i \quad(p \text { prime })
$$

we have

$$
\operatorname{ord}_{N}\left(\begin{array}{c}
k \\
i
\end{array}\right) \geq a-\max _{p \mid i}\left(\operatorname{ord}_{p} i\right) .
$$

It follows that

$$
\operatorname{ord}_{N}\left(\left(\begin{array}{c}
k \\
i
\end{array}\right) N^{i}\right) \geq a-\max _{\substack{p \mid i \\
p \text { odd }}}\left(\operatorname{ord}_{p} i\right)+i
$$

and so if $i \geq 2$,

$$
\operatorname{ord}_{N}\left(\left(\begin{array}{c}
k \\
i
\end{array}\right) N^{i}\right) \geq a+2 .
$$

We conclude, then, that $N^{a+1} \mid k$ as required and hence (6) has no solutions for $k$ odd.

We will also use

Lemma 2.2. If $n$ and $l$ are integers with $n>l \geq(N+1)^{k}$, then there is an element of $S_{N}^{(k)}$, say $i^{k}$, such that

$$
l \leq n-i^{k}<l+k n^{(k-1) / k} .
$$


Pr o o f. Suppose first that $n \geq l+N^{k}$ and choose $i$ such that $i^{k} \leq n-l<$ $(i+1)^{k}$. Then $i^{k} \in S_{N}^{(k)}$ and since, by calculus,

$$
n-l-i^{k} \leq k(n-l)^{(k-1) / k}<k n^{(k-1) / k},
$$

we have (7). If, however, $n<l+N^{k}$, take $i=1$ and write $n=l+m$ (so that $\left.1 \leq m<N^{k}\right)$. We conclude

$$
k(l+m)^{(k-1) / k}>k(N+1)^{k-1}=\frac{k}{N+1}(N+1)^{k} .
$$

Since $k \geq N+1$, this is at least $(N+1)^{k}$ and hence greater than $m$, as desired.

Let us now begin to consider representations of comparatively small integers as sums of elements of $S_{N}^{(k)}$. We have

Lemma 2.3. $\left[1, \alpha N^{k}\right] \in S_{N}^{(k)}\left(I_{N}^{(k)}\right)$ where $I_{N}^{(k)}=N^{k}+\alpha-2$.

Proof. If $M \leq \alpha N^{k}-1$, then we can write $M=N^{k} x+y$ with $0 \leq$ $y \leq N^{k}-1$ and $x<\alpha$. It follows that $M$ is a sum of $x+y \leq N^{k}+\alpha-2$ elements of $S_{N}^{(k)}$. If, however, $M=\alpha N^{k}$, clearly $M \in S_{N}^{(k)}(\alpha)$.

LEMma 2.4. $\left(\alpha N^{k},(\alpha+1) N^{k}\right) \in S_{N}^{(k)}(E)$ where $E=\max \{\alpha+\beta-1$, $\left.N^{k}-\beta\right\}$.

Proof. The integers $\alpha N^{k}, \alpha N^{k}+1, \ldots, \alpha N^{k}+\beta-1$ are in $S_{N}^{(k)}(\alpha+\beta-1)$ while $\alpha N^{k}+\beta=(N+1)^{k}, \ldots, \alpha N^{k}+N^{k}-1=(N+1)^{k}-\beta+N^{k}-1$ belong to $S_{N}^{(k)}\left(N^{k}-\beta\right)$. Since $(\alpha+1) N^{k} \in S_{N}^{(k)}(\alpha+1)$ and $\beta \geq 2$ via Lemma 2.1, we are done.

The beginning of our ascent argument, following Dickson [6], lies in

LEMMA 2.5. If $p$ and $L$ are positive integers with $p \geq N$ and $(L, L+$ $\left.p^{k}\right) \in S_{N}^{(k)}(m)$, then $\left(L, L+2 p^{k}\right) \in S_{N}^{(k)}(m+1)$.

Proof. Let $M$ be an integer satisfying

$$
L+p^{k} \leq M<L+2 p^{k} .
$$

Then $M-p^{k} \in S_{N}^{(k)}(m)$ and so $M \in S_{N}^{(k)}(m+1)$. If $M \in\left(L, L+p^{k}\right)$, the result is trivial.

By induction on $n$, we readily obtain

Lemma 2.6. If $p, n$ and $L$ are positive integers with $p \geq N$ and $(L, L+$ $\left.p^{k}\right) \in S_{N}^{(k)}(m)$, then $\left(L, L+p^{k}(n+1)\right) \in S_{N}^{(k)}(m+n)$.

Taking $L=\alpha N^{k}, p=N, n=\alpha+1$ and applying Lemmas 2.4 and 2.6 we conclude, from $n N^{k}>(N+1)^{k}$,

LEMma 2.7. $\left(\alpha N^{k}, \alpha N^{k}+(N+1)^{k}\right) \in S_{N}^{(k)}(E+\alpha)$. 
If we now successively apply Lemma 2.7 and Lemma 2.6 with $p=N+1$, $N+2, \ldots, k$ and

$$
n=\left[\left(\frac{N+2}{N+1}\right)^{k}\right],\left[\left(\frac{N+3}{N+2}\right)^{k}\right], \ldots,\left[\left(\frac{k+1}{k}\right)^{k}\right],
$$

it follows that

LEMMA 2.8 .

$\left(\alpha N^{k}, \alpha N^{k}+(k+1)^{k}\right) \in S_{N}^{(k)}\left(E+\alpha+\left[\left(\frac{N+2}{N+1}\right)^{k}\right]+\ldots+\left[\left(\frac{k+1}{k}\right)^{k}\right]\right)$.

Our main ascent relies upon the following result, which is essentially a variant of a theorem of Dickson [5, Theorem 12].

Proposition 2.9. Let $l$ and $L_{0}$ be integers with

$$
L_{0}>l \geq(N+1)^{k}, \quad v=\left(1-l / L_{0}\right) / k \quad \text { and } \quad v^{k} L_{0} \geq 1 .
$$

If for $t \in \mathbb{N}$ we define $L_{t}$ by

$$
\log L_{t}=\left(\frac{k}{k-1}\right)^{t}\left(\log L_{0}+k \log v\right)-k \log v
$$

and if $\left(l, L_{0}\right) \in S_{N}^{(k)}(m)$, then $\left(l, L_{t}\right) \in S_{N}^{(k)}(m+t)$.

Proof. We suppose $\left(l, L_{0}\right) \in S_{N}^{(k)}(m)$ and that $n \in\left(l, L_{1}\right)$. Now for $t=1$, (8) is equivalent to

$$
L_{1}=\left(v L_{0}\right)^{k /(k-1)}
$$

and hence we may use Lemma 2.2 to find $i^{k} \in S_{N}^{(k)}$ such that

$$
l \leq n-i^{k}<l+k n^{(k-1) / k}<l+k v L_{0} .
$$

Since $v=\left(1-l / L_{0}\right) / k$, we have $l \leq n-i^{k}<L_{0}$, whence $\left(l, L_{1}\right) \in S_{N}^{(k)}(m+1)$. In general, (8) yields

$$
L_{t+1}=\left(v L_{t}\right)^{k /(k-1)}
$$

and the result obtains by induction upon $t$.

3. Proof of Theorem 1.1. Assume $N \geq 4$. To apply the preceding proposition, we let $l=(N+1)^{k}$ and $L_{0}=(k+1)^{k}$. The condition that $v^{k} L_{0} \geq 1$ is then equivalent to

$$
N \leq(k+1)^{(k-1) / k}-1 .
$$

If we choose $t$ large enough that

$$
L_{t}>\max \left\{N^{8 k^{3}}, e^{446 k^{6}}\right\}=e^{446 k^{6}}
$$


then Theorem 1.2 gives $\left[L_{t}, \infty\right) \in S_{N}^{(k)}(6 k \log k+(3 \log 6+4) k)$. Now from $v=\left(1-l / L_{0}\right) / k$, we may write

$$
\begin{aligned}
\log L_{t} & =\left(\frac{k}{k-1}\right)^{t}(k \log (k+1)-k \log v)-k \log v \\
& >\left(\frac{k}{k-1}\right)^{t}\left(k \log \left(\frac{k+1}{k}\right)\right) .
\end{aligned}
$$

Since

$$
\log \left(\frac{k+1}{k}\right)>\frac{1}{k}-\frac{1}{2 k^{2}} \geq \frac{11}{12 k} \quad \text { for } k \geq 6,
$$

this implies

$$
\log L_{t}>\frac{11}{12}\left(\frac{k}{k-1}\right)^{t}
$$

If we note that

$$
\log \left(\frac{k}{k-1}\right)>\frac{1}{k-1}-\frac{1}{2(k-1)^{2}}>\frac{1}{k}
$$

we obtain (9) provided

$$
t>k\left(6 \log k+\log \left(\frac{5352}{11}\right)\right) .
$$

Taking $t=[6 k \log k+7 k]$, then, yields the desired conclusion. By Lemma 2.3, it remains to show for this choice of $t$ that $\left(\alpha N^{k}, L_{t}\right) \in S_{N}^{(k)}\left(I_{N}^{(k)}\right)$ (we have $\left[L_{t}, \infty\right) \in S_{N}^{(k)}\left(I_{N}^{(k)}\right)$ because $6 k \log k+(3 \log 6+4) k<I_{N}^{(k)}$ for $4 \leq N \leq$ $\left.(k+1)^{(k-1) / k}-1\right)$.

By Lemma 2.8 and Proposition 2.9, we have

$$
\left(\alpha N^{k}, L_{t}\right) \in S_{N}^{(k)}\left(E+\alpha+t+(k-N)\left[\left(\frac{N+2}{N+1}\right)^{k}\right]\right)
$$

and this follows from

$$
E+\alpha+t+(k-N)\left[\left(\frac{N+2}{N+1}\right)^{k}\right] \leq I_{N}^{(k)}=N^{k}+\alpha-2 .
$$

If $E=\alpha+\beta-1$, then (10) becomes

$$
\alpha+\beta+t+(k-N)\left[\left(\frac{N+2}{N+1}\right)^{k}\right]-N^{k} \leq-1
$$

while $E=N^{k}-\beta$ implies the inequality

$$
t+(k-N)\left[\left(\frac{N+2}{N+1}\right)^{k}\right]-\beta \leq-2 .
$$


To prove that (11) and (12) obtain for all $N$ and $k$ satisfying

$$
4 \leq N \leq(k+1)^{(k-1) / k}-1
$$

we employ Theorem 1.3 to deduce

$$
3^{-k}<\beta / N^{k}<1-3^{-k} \text {. }
$$

The left hand side of (11) is then bounded above by

$$
\left(\frac{N+1}{N}\right)^{k}-\left(\frac{N}{3}\right)^{k}+6 k \log k+7 k+(k-N)\left(\frac{N+2}{N+1}\right)^{k}
$$

and hence is $\leq-1$ for $N$ and $k$ unless

(i) $N=4,6 \leq k \leq 34$, or

(ii) $N=5,8 \leq k \leq 11$.

Additionally, we bound the left hand side of (12) by

$$
6 k \log k+7 k+(k-N)\left(\frac{N+2}{N+1}\right)^{k}-\left(\frac{N}{3}\right)^{k},
$$

which is $\leq-2$ for all values of $N$ and $k$ under consideration except

(iii) $N=4,6 \leq k \leq 32$, and

(iv) $N=5,8 \leq k \leq 11$.

Checking that (11) and (12) hold for the cases (i), (ii) and (iii), (iv) respectively, we conclude the proof of the theorem by noting that $M=$ $\alpha N^{k}-1 \notin S_{N}^{(k)}\left(N^{k}+\alpha-3\right)$ and thus

$$
N^{k}+\left[\left(\frac{N+1}{N}\right)^{k}\right]-2 \leq g_{N}(k) \leq N^{k}+\left[\left(\frac{N+1}{N}\right)^{k}\right]-2 \text {. }
$$

4. Concluding remarks. If $N=3$ and $k \geq 6$, we can show that

$$
g_{3}(k)=3^{k}+\left[(4 / 3)^{k}\right]-2
$$

provided

$$
\left\|(4 / 3)^{k}\right\|>(9 / 4)^{-k}
$$

(in general, we require only

$$
\left\|\left(\frac{N+1}{N}\right)^{k}\right\|>\left(\frac{N^{2}}{N+1}\right)^{-k},
$$

which is rather weaker than Theorem 1.3). Though we have (13) for all but finitely many $k$ by Mahler's result, it seems difficult to prove effective bounds approaching the above in strength (see Baker and Coates [1] for the only known nontrivial bound in this situation). As mentioned previously, 
the case $N=2$ (the ideal Waring problem) also remains open. The best effective result for $\left\|(3 / 2)^{k}\right\|$ is due to Dubitskas, who proved

TheOREM 4.1 (Dubitskas [7]). There is an effectively computable $k_{0}$ such that if $k \geq k_{0}$, then

$$
\left\|(3 / 2)^{k}\right\|>(1.734)^{-k} .
$$

Unfortunately, this falls rather short of the desired lower bound of $(4 / 3)^{-k}$.

\section{References}

[1] A. Baker and J. Coates, Fractional parts of powers of rationals, Math. Proc. Cambridge Philos. Soc. 77 (1975), 269-279.

[2] M. Bennett, Fractional parts of powers and related topics, Ph.D. thesis, University of British Columbia, 1993.

[3] - Fractional parts of powers of rational numbers, Math. Proc. Cambridge Philos. Soc. 114 (1993), 191-201.

[4] F. Beukers, Fractional parts of powers of rationals, ibid. 90 (1981), 13-20.

[5] L. E. Dickson, Recent progress on Waring's theorem and its generalizations, Bull. Amer. Math. Soc. 39 (1933), 701-702.

[6] - Proof of the ideal Waring's theorem for exponents 7-180, Amer. J. Math. 58 (1936), 521-529.

[7] A. K. Dubitskas, A lower bound for the quantity $\left\|(3 / 2)^{k}\right\|$, Russian Math. Surveys 45 (1990), 163-164.

[8] J. M. Kubina and M. C. Wunderlich, Extending Waring's conjecture to 471,600,000, Math. Comp. 55 (1990), 815-820.

[9] K. Mahler, On the fractional parts of the powers of a rational number: II, Mathematika 4 (1957), 122-124.

[10] G. J. Rieger, Über eine Verallgemeinerung des Waringschen Problems, Math. Z. 58 (1953), 281-283.

[11] I. M. Vinogradov, On Waring's problem, Ann. of Math. 36 (1935), 395-405.

DEPARTMENT OF PURE MATHEMATICS

UNIVERSITY OF WATERLOO

WATERLOO, ONTARIO

CANADA N2L 3G1

E-mail: MABENNETT@JEEVES.UWATERLOO.CA 